\title{
APP MIDOAA: Objeto de Aprendizagem Acessível para Apoiar Estudantes com Deficiência Auditiva
}

\author{
Andreza Bastos Mourão ${ }^{1,2}$, Christian Bezerra de Menezes ${ }^{3}$, Arcanjo Miguel Mota \\ Lopes $^{1}$, José Francisco de Magalhães Netto ${ }^{1}$ \\ ${ }^{1}$ Programa de Pós-Graduação em Informática (PPGI) - Universidade Federal do \\ Amazonas (UFAM) \\ Av. Gen. Rodrigo Otávio, Coroado. Manaus - AM - Brasil \\ ${ }^{2}$ Universidade do Estado do Amazonas (UEA) \\ Av. Darcy Vargas, 1200. Manaus - AM - Brasil \\ ${ }^{3}$ Uniasselvi - Centro Universitário Leonardo da Vinci - Pólo Itacoatiara \\ Itacoatiara, AM - Brasil \\ amourao@uea.edu.br, christian menezes@hotmail.com, \\ amml@icomp.ufam.edu.br, jnetto@icomp.ufam.edu.br
}

\begin{abstract}
Resumo. Este white paper faz a descrição suscinta de um Objeto de Aprendizagem Acessível para Computação, embarcado em um Aplicativo móvel que tem como público-alvo estudantes com deficiência auditiva matriculados em cursos de nível superior na área da computação. O objetivo é apoiar, incentivar e contribuir com Professores de Ensino Superior em Computação, a inovar suas práticas pedagógicas e possibilitar um olhar e a realizações de práticas inclusivas. O App foi desenvolvido na plataforma Visual Class, atende a padrões e diretrizes de acessibilidade, está disponível nas plataformas Móvel e Desktop, possui um tradutor de Libras e ao final gera um relatório de notas.
\end{abstract}

\section{Cenário de uso}

A educação é um direito previsto por lei que garante o ensino, acesso e participação de todas as pessoas nas escolas e universidades em todos os níveis de ensino [Mourão e Netto 2018a]. Neste contexto, a educação deve assegurar aos estudantes, independente de suas limitações, exercer seus direitos fundamentais perante a sociedade. As instituições de ensino devem prover mecanismos, ferramentas, recursos e projetos voltados para atender estudantes com deficiência, ou com algum tipo de transtorno, como por exemplo: Transtorno do Déficit de Atenção com Hiperatividade (TDAH), Dislexia, Discalculia entre outros.

O problema geral é que o mundo ainda não está preparado para lidar com a acessibilidade. Alguns países mais desenvolvidos já investem em infraestrutura e recursos educacionais para atender necessidades reais [Mourão e Netto 2018b]. No entanto, o Brasil ainda está engatinhando neste contexto apesar de estar em vigor a legislação [Brasil 2019] que promove a inclusão de Pessoas com Deficiência (PCD), cujo objetivo é promover inclusão e cidadania de forma igualitária. 
Este panorama desencadeou a motivação pela busca e desenvolvimento de uma solução tecnológica que pudesse atender uma necessidade atual identificada em cursos superiores de computação de instituições públicas e privadas na cidade de Manaus no Estado do Amazonas. A identificação e viabilidade do recurso educacional foram possíveis mediante a aplicação de um questionário de viabilidade com professores e coordenadores de instituições públicas e privadas de ensino [Mourão e Netto 2018a].

Nos dias atuais professores e gestores guiam suas ações e intervenções, contemplando o uso das Novas Tecnologias Digitais da Informação e Comunicação (NTDIC) como recursos potencializadores da aprendizagem. Siqueira (2015) afirma que "apesar da inclusão escolar ser uma realidade na legislação brasileira, verifica-se no cotidiano das instituições de ensino regular, particulares e públicas, certa dificuldade em lidar com a adequação às demandas dos alunos especiais". É neste sentido, que os modelos inclusivos vêm se consolidando e gerando contribuições significativas neste cenário educacional.

Considerando o cenário acima e práticas já desenvolvidas por Mourão e Netto (2016), (2018b) e (2019), optou-se pelo desenvolvimento de um Objeto de Aprendizagem Acessível que atende ao Modelo Inclusivo proposto por Mourão e Netto (2018a), visando promover o aprendizado móvel $^{1}$ (M-learning) e presencial (Desktop).

Nos dias atuais professores e estudantes utilizam dispositivos móveis para acessar e compartilhar conteúdos educacionais, colaborar e construir conhecimento, facilitando desta forma a comunicação entre pares e grupos. Dentre os principais benefícios do aplicativo destacam-se: promover uma prática inovadora e inclusiva, incentivar um ambiente igualitário e a educação acadêmica ${ }^{2}$, favorecer um ambiente socioemocional e produzir impacto positivo nos resultados acadêmicos.

O aplicativo foi desenvolvido no software de autoria Visual Class, tendo como público-alvo estudantes sem e com deficiência auditiva, matriculados em cursos superiores de Computação. A aplicação foi validada por estudantes do Curso de Sistema de Informação, na disciplina de Introdução a Programação de Computadores, onde a linguagem de programação ensinada foi Phyton, e cuja unidade instrucional selecionada e trabalhada foi Arquivos em Python.

\section{Desenvolvimento}

O APP MIDOAA é o primeiro aplicativo de muitos outros que serão desenvolvidos com a finalidade de apoiar atividades educacionais no contexto da educação inclusiva. $O$ desenvolvimento do OAA na plataforma móvel faz parte do projeto MIDOAA [Mourão e Netto 2018a].

O processo adotado para o desenvolvimento do aplicativo seguiu as etapas definidas do Modelo Inclusivo MIDOAA proposto por Mourão e Netto (2018a). O desenvolvimento do OAA utilizou as abordagens pedagógica e computacional. O processo foi definido utilizando a metodologia PDCA (Planejar, Executar, Verificar e Agir), conforme mostrado na Figura 1.

\footnotetext{
1 A aprendizagem móvel refere-se ao processo de aprendizagem mediado por tecnologias móveis, como smartphones, tablets e consoles de videogame [Schuler, Winters, e West, 2012].

${ }^{2}$ Educação acadêmica refere-se ao número de anos de educação que um indivíduo completa (Schifter, 2015).
} 
VIII Congresso Brasileiro de Informática na Educação (CBIE 2019)

Anais dos Workshops do VIII Congresso Brasileiro de Informática na Educação (WCBIE 2019)

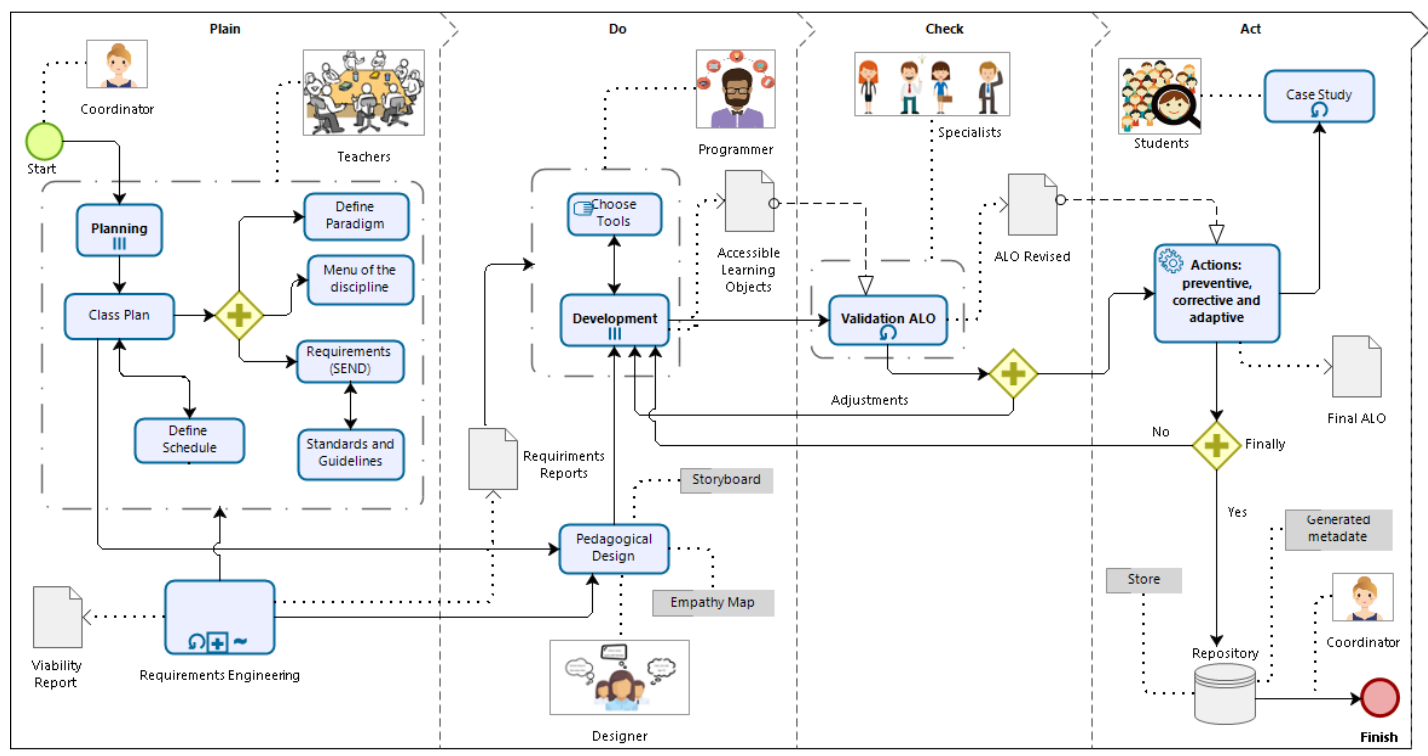

Figura 1 - Processo do Modelo Inclusivo MIDOAA [Mourão e Netto 2018]

As quatro etapas foram seguidas, definidas e desenvolvidas como descritas a seguir:

1 - Planejar: nesta etapa, foram utilizados conceitos, técnicas e ferramentas da Engenharia de Requisitos. Inicialmente foi realizado um estudo de viabilidade do App que gerou um relatório (contendo custo, risco e viabilidade de desenvolvimento do projeto). Em seguida, na etapa de elicitação de requisitos foram utilizadas as técnicas de questionário, entrevista e mapa de empatia. $\mathrm{O}$ questionário e a entrevista possibilitaram conhecer mais sobre a turma, a disciplina, o conteúdo instrucional, permitindo assim, definir os objetivos pedagógicos e computacionais; os requisitos de aprendizagem, usabilidade e acessibilidade; a deficiência a ser atendida; infraestrutura; tecnologias utilizadas; metodologia; padrões e diretrizes acessíveis; e definir o cronograma de desenvolvimento, entrega e avaliação. O mapa de empatia foi aplicado na turma com todos os estudantes e com o professor da turma com o objetivo de desenvolver o persona do estudante com deficiência auditiva e outro persona que representasse os demais estudantes da classe. Desta forma, foi possível criar um protótipo de OAA voltado para atender as características apresentadas no Mapa (Figura 2).
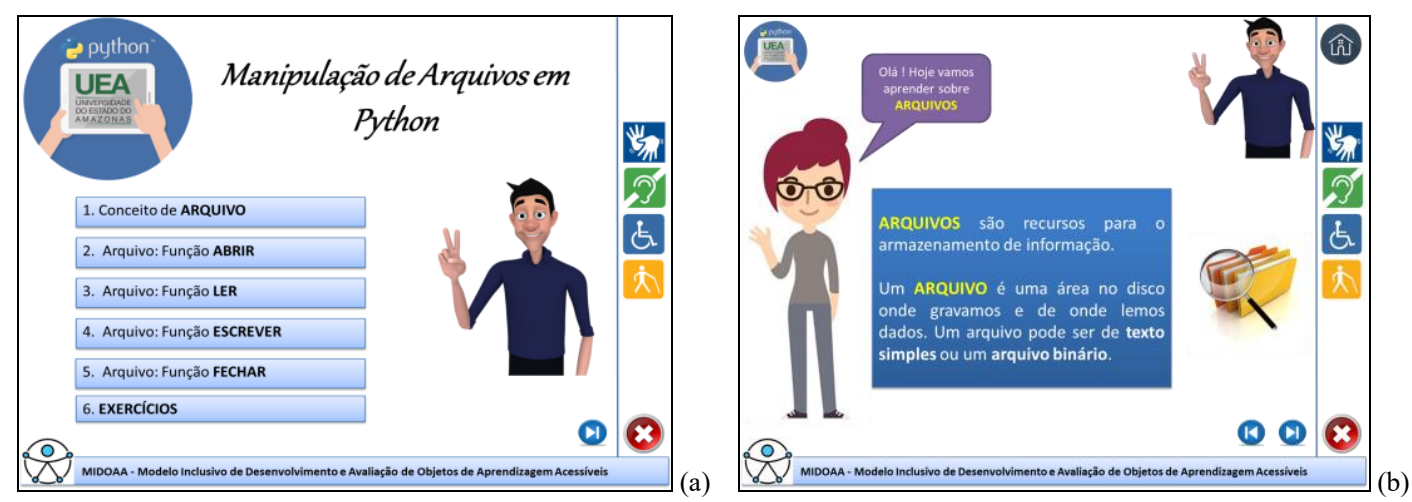

Figura 2 - Protótipo do OAA - (a) Tela Principal / (b) Segunda Tela. 
2 - Executar: nesta etapa, foi utilizado como parâmetro o relatório de requisitos funcionais e não funcionais gerados na etapa anterior que serviram como base para o desenvolvimento do aplicativo. O App foi desenvolvido por um professor da área da computação e por um técnico da área de informática na educação especialista no software de autoria Visual Class, Class Player, Class Net e Visual Class Net, e com todo o suporte da Caltech Informática $\mathrm{Ltda}^{3}$. O App atende a um design pedagógico (com base no Mapa de Empatia e Modelo Navegacional) e a padrões e diretrizes de acessibilidade.

O Aplicativo móvel do OAA é multiplataforma Android (acessando o Google Play), IOS (acessando a Apple Store) podendo ser baixado gratuitamente. $\mathrm{O}$ acesso também pode ocorrer via desktop, por meio do Sistema Operacional Windows ou macOS. Para viabilizar o uso pode-se utilizar o software gratuito Nox Player4 que permite emular com perfeição o S.O. Android em PC ou Notebook. Depois de instalado o emulador, basta configurar uma conta para acessar o Google Play ou o AppStore.

O APP MIDOAA foi desenvolvido utilizando as seguintes tecnologias:

- Tecnologias gratuitas: DSpeech (conversão de texto em áudio);

- Tecnologias pagas: Software de Autoria Visual Class, Class Player (tocador de projetos do Visual Class), a Universidade (UEA) é parceira e possui licença. Software Ivona (conversão de texto em áudio), Software Photoshop (Criação e Edição de Imagens) e Software Gravador de Tela Pro da Apowersoft (Captura e edição dos vídeos em Libras).

3 - Verificar: nesta etapa, considerou-se o protótipo modelo como parâmetro para validar as funcionalidades do OAA, assim como o relatório gerado na primeira fase contendo os requisitos funcionais e não funcionais de aprendizagem, acessibilidade e usabilidade. Para a validação do aplicativo foram considerados os protótipos, os personas gerados por meio dos mapas de empatias aplicados com a turma e os relatórios gerados pelas etapas da Engenharia de Requisitos que deram suporte do inicio até o final do processo.

4 - Agir: nesta etapa, foram aplicados questionários com os estudantes da turma que utilizaram o App, visando avaliar o OAA quanto às abordagens de ensino, aprendizagem, usabilidade e acessibilidade. A avaliação e ajustes finais do produto APP também teve a participação de especialistas, professores e técnicos. A avaliação foi realizada por meio de questionários produzidos e disponibilizados para esta finalidade e disponível na plataforma Google Forms. Após a avaliação final, os resultados foram tabelados e serviram como base para correções e ajustes finais, considerando as limitações apresentadas pela ferramenta. Os itens que apresentaram o maior índice de aceitação e que são primordiais no processo de avaliação de aplicativos, expressaram resultados animadores como: desempenho e tempo de resposta; recursos e funcionalidade; confiabilidade, aparência, usabilidade, acessibilidade e navegação; e segurança. Desta forma, o OAA está disponível na Internet para ser utilizado.

\footnotetext{
3 Disponível em: http://www.classinformatica.com.br/

4 Disponível em: https://pt.bignox.com/
} 
VIII Congresso Brasileiro de Informática na Educação (CBIE 2019)

Anais dos Workshops do VIII Congresso Brasileiro de Informática na Educação (WCBIE 2019)

\section{Apresentação do Software}

O aplicativo App MIDOAA é gratuito, e foi desenvolvido para apoiar a educação inclusiva no ensino superior, faz parte de uma das inúmeras ações que vem sendo desenvolvidas ao longo dos três últimos anos. Este aplicativo faz parte do projeto MIDOAA [Mourão e Netto 2018], foi desenvolvido para atender um público-alvo específico, unidade instrucional, disciplina e turma, visando comprovar o processo, diretrizes e padrões definidos pelo MIDOAA.

O design, modelo navegacional, processo e a tecnologia foram fatores determinantes para a entrega e disponibilidade do produto. $\mathrm{O}$ app atende estudantes não deficientes e com deficiência física, podendo ser utilizado em outras situações. A partir deste modelo, pretende-se expandir o projeto a fim de viabilizar o desenvolvimento de muitos outros aplicativos para atender inúmeras outras deficiências e requisitos.

O APP MIDOAA é um aplicativo móvel que pode ser baixado no Play Store ou na APP Store. Os passos abaixo demonstram como os aplicativos podem ser acessados em cada uma das plataformas.

1. App Store - O aplicativo por ser baixado e acessado por iPhone - faça o download na App Store seguindo este procedimento:

a) Entre na App Store clicando no ícone da App Store no iPhone;

b) Na App Store, pesquise por "Class Player" e baixe o APP;

c) Após achar o aplicativo, clique em "Obter";

d) Em seguida, clique em "Instalar";

e) Insira um ID e senha Apple válidos;

f) Após inserir a senha, o aplicativo Class Player será baixado e instalado;

g) Após o download, você poderá encontrar o aplicativo na tela do dispositivo móvel - toque para abrir o aplicativo Class Player;

2. Play Store - O aplicativo por ser baixado e acessado por dispositivos Android faça o download na Google Play Store seguindo este procedimento:

a) Entre no Google Play tocando no ícone Play Store em seu telefone Android;

b) No Google Play, pesquise por "Class Player" e baixe o APP;

c) Ao encontrar o aplicativo, toque em "Instalar", seguido de "Aceitar", para permitir que o aplicativo acesse determinados recursos do seu telefone, como o Bluetooth;

d) Insira um ID e senha Google (gmail) válidos;

e) Após inserir a senha, o aplicativo Class Player será baixado e instalado;

f) Ao fim da instalação, toque em "Abrir" para abrir o aplicativo "Class Player";

g) Após o download, você poderá encontrar o aplicativo na tela do dispositivo móvel - toque para abrir o aplicativo Class Player;

Após a execução dos passos listados acima em cada uma das plataformas. É possível baixar o aplicativo MIDOAA, por meio da interface do Class Player. Em seguida, baixe o APP, click no Menu senha (Figura 3a), digite MIDOAA ou midoaa e click no botão OK. Em seguida, encontrará o ícone MIDOAA UEA, então é só dar um click no botão instalar (Figura 3b). Depois de instalado não precisará mais de acesso a internet e poderá utilizá-lo off-line. 
VIII Congresso Brasileiro de Informática na Educação (CBIE 2019)

Anais dos Workshops do VIII Congresso Brasileiro de Informática na Educação (WCBIE 2019)
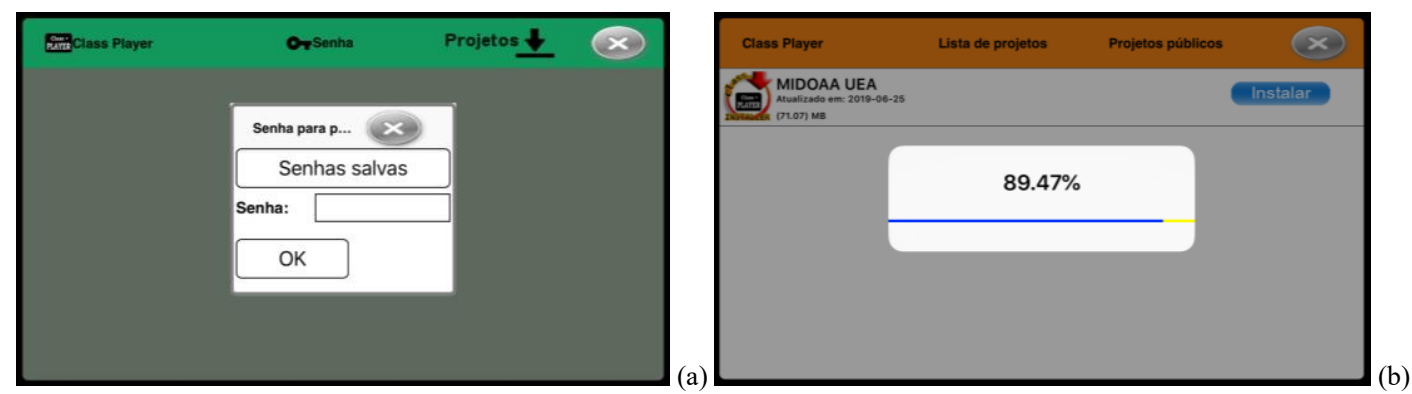

Figura 3 - Interface do App Class Player - (a) Tela Senha / (b) Tela de Download

A Figura 4(a) mostra a interface do Class Player com o ícone do MIDOAA instalado após o download. A Figura 4(b) mostra a tela de inicial do MIDOAA. O usuário ao dar um click no botão iniciar no canto inferior direito (Figura 4b), terá acesso ao menu principal do Aplicativo.
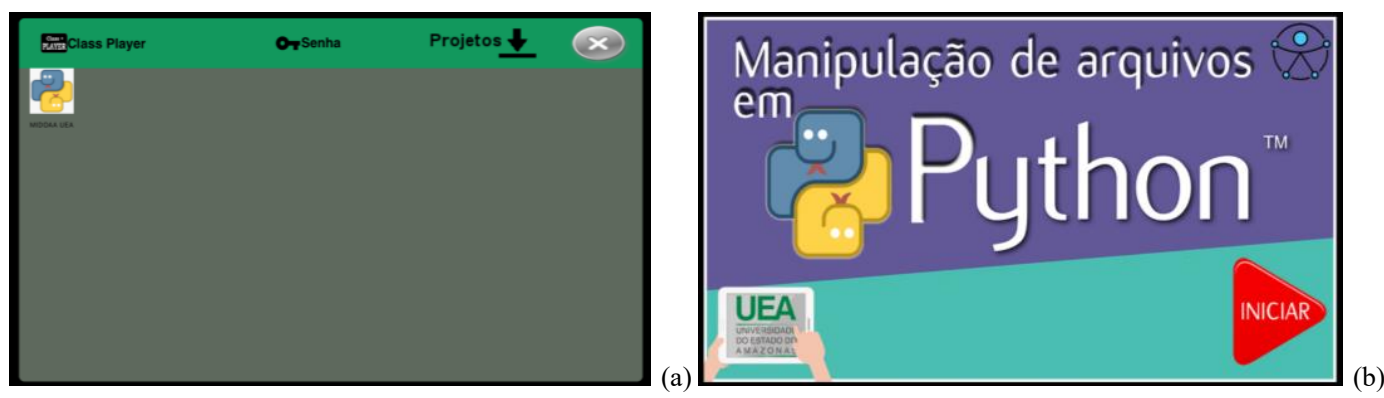

Figura 4 - Interface do App Class Player - (a) Tela do Class Player com App MIDOAA UEA / (b) Tela Inicial do App

A Figura 5 apresenta a interface principal do Aplicativo. A Interface principal além do Menu (-), possui o personagem animado em Libras da ProDeaf ${ }^{5}$ (presente em todo o App), as referências, os ícones de acessibilidade, a logomarca da instituição de ensino superior e o botão de navegação (Sair).

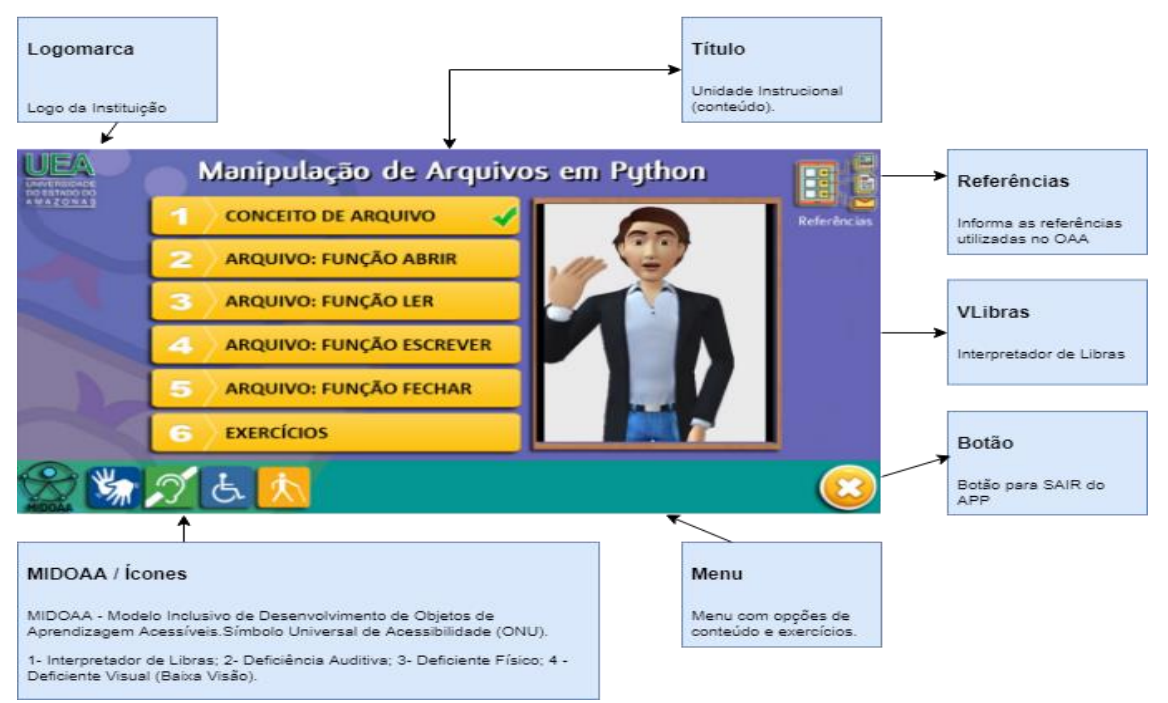

Figura 5 - Interface do Menu principal do App MIDOAA

\footnotetext{
${ }^{5}$ A ProDeaf é o primeiro aplicativo tradutor para a Língua Brasileira de Sinais. Atualmente se uniu à Hand Talk para levantar a bandeira da acessibilidade em Libras [ProDeaf, 2018].
} 
VIII Congresso Brasileiro de Informática na Educação (CBIE 2019)

Anais dos Workshops do VIII Congresso Brasileiro de Informática na Educação (WCBIE 2019)

As informações estão detalhadas ao redor da Figura 5. É importante destacar que o aplicativo atende os deficientes auditivos, e deficientes físico e visual com nível de deficiência de aproximado de $25 \%$ (moderado a leve).

É importante destacar os botões de navegação (Figura 6a), onde o usuário pode voltar @ ou avançar @ uma tela, ou pode optar por Sair @ do App. A Figura 6b apresenta a interface do conteúdo: Função Abrir em Python, nesta tela é possível acessar o vídeo aula retorna a Tela. O usuário pode Sair ou Voltar para o Menu principal, é só dar um click na opção desejada.
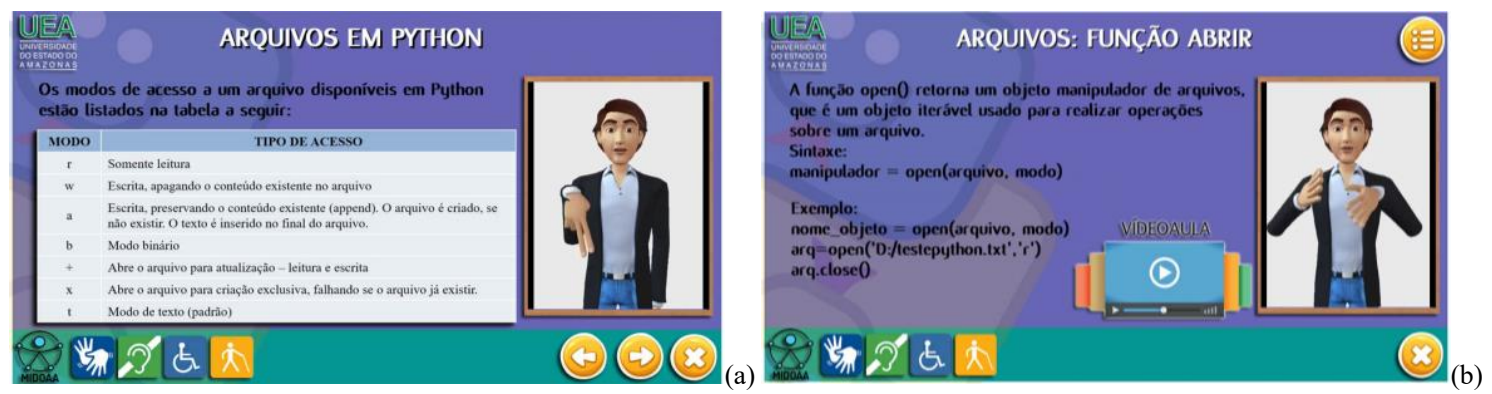

Figura 6 - Interface do App MIDOAA - Tela Arquivos: Função Abrir

Os estudantes podem realizar seus exercícios, conforme exemplo mostrado na Figura 7(a). Estão disponíveis 5 tipos de exercícios avaliativos, os estudantes respondem com base no estudo prévio. Ao finalizar, o estudante retorna ao Menu

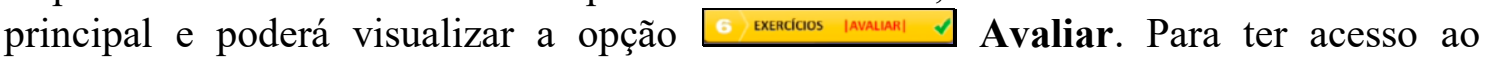
resultado o estudante dá um click e então obtém o feedback da sua avaliação (Figura $7 b$ ), contendo a data, tempo, sua nota, a quantidade de erros e acertos e resumo.
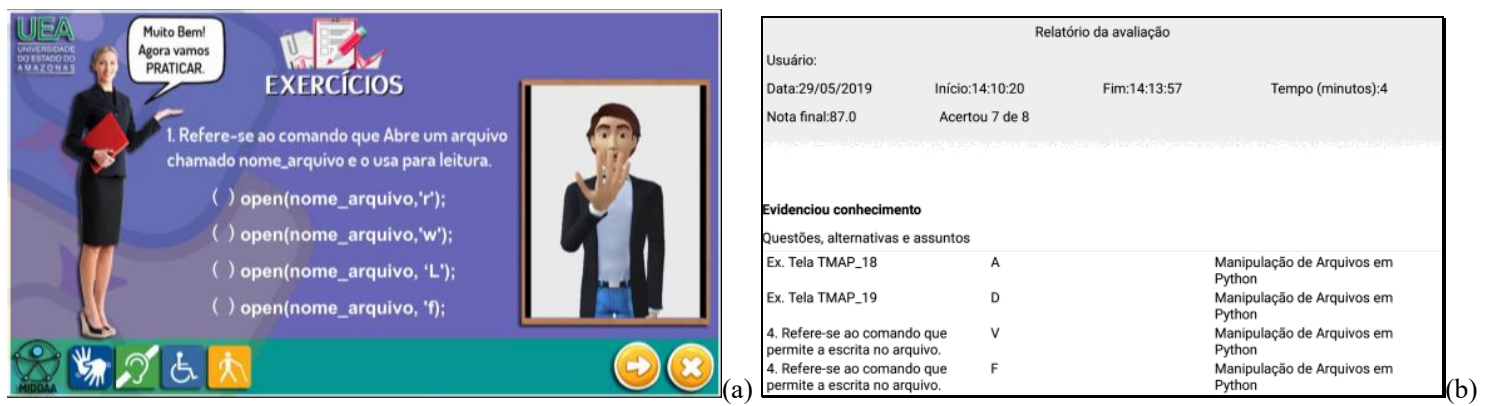

Figura 7 - Interface do App MIDOAA - Tela Exercícios - (a) Tela do Exercício 1 / (b) Tela do Relatório de Aprendizagem do Estudante

O vídeo demonstrativo encontra-se disponível no Youtube ${ }^{6}$, o APP faz parte do projeto MIDOAA disponível na página da Universidade (http://midoaa.uea.edu.br/midoaa/appmidoaa).

\footnotetext{
${ }^{6}$ Disponível em: https://www.youtube.com/watch?v=kVvL4NHGeBA
} 
VIII Congresso Brasileiro de Informática na Educação (CBIE 2019)

Anais dos Workshops do VIII Congresso Brasileiro de Informática na Educação (WCBIE 2019)

\section{Considerações finais}

O APP MIDOAA é um aplicativo para apoiar o ensino da Linguagem de Programação Python, em cursos de nível superior em Computação, para estudantes com deficiências. Existe um número expressivo de estudantes com deficiências buscando qualificação superior, e projetos de inclusão como o aplicativo MIDOAA, os auxiliam a atingir esse resultado.

O aplicativo contribui para a Educação Inclusiva, permitindo o aprendizado de "Python" por estudantes com Deficiência Auditiva, apresentando todo o conteúdo de forma sequencial, seguindo uma abordagem pedagógica e computacional. O APP é baseado na plataforma Visual Class e as aulas são traduzidas por um personagem animado em Libras.

O aplicativo foi validado por meio de um estudo de caso, no Curso de Sistemas de Informação, na turma do $1^{\circ}$ período, na disciplina de Introdução a Programação de Computadores da EST/UEA. A turma foi escolhida, pois havia um estudante com deficiência auditiva, regularmente matriculado, e assim promoveu-se um processo de ensino e aprendizagem inclusivo. Em seguida, todos os estudantes utilizaram o Aplicativo e avaliaram (por meio de formulário), que demonstrou impacto positivo, aceitabilidade, originalidade, facilidade de navegação e usabilidade. O APP promoveu o aprendizado e o desenvolvimento socioemocional de todos os estudantes estimulando positivamente o relacionamento entre eles.

Como perspectivas futuras, pretende-se criar visibilidade e expandir o projeto APP MIDOAA para o desenvolvimento de novos aplicativos que possam apoiar a aprendizagem móvel na área da computação e posteriormente em outras áreas do ensino superior. Os aplicativos desenvolvidos serão armazenados e compartilhados no repositório SIMROAA $^{7}(2019 b)$. Em paralelo almejamos criar parcerias com pesquisadores de outras Universidades para ampliarmos a produção e 0 compartilhamento de APP, visando atender as diversas deficiências e transtornos apresentados por estudantes de nível superior.

\section{Referências}

Brasil. Decreto $\mathrm{n}^{\mathrm{o}}$ 13.146, 6 de Julho de 2015. (2019). Dispõe sobre a Lei Brasileira de Inclusão de Pessoas com Deficiência. Disponível em: http://www.planalto.gov.br/ccivil_03/_ato2015-2018/2015/lei/113146.htm. Acessado em: 11 de Junho de 2019.

Mourão, Andreza B., Netto, José Francisco. (2018a). MIDOAA: Inclusive Model of Development of Accessible Learning Objects. In: FIE 2018 - 48th Annual Frontiers In Education Conference, 2018, San Jose, California.

Mourão, Andreza B., Netto, José Francisco. (2018b). Inclusive Model for the Development and Evaluation of Accessible Learning Objects for graduation in Computing: A Case Study. In: FIE 2018, San Jose, California.

Mourão, Andreza Bastos \& Netto, José Francisco de Magalhães. (2019a). Inclusive Model Application Using Accessible Learning Objects to Support the Teaching of Mathematics. Informatics in Education. 18. 213-226.

Mourão, Andreza B., Netto, José Francisco. (2019b). SIMROAA: Multi-Agent Recommendation System for Recommending Accessible Learning Objects. In: FIE 2019, Cincinnati, Ohio.

Siqueira, Maria das Graças Soares; Aguillera, Fernanda. Modelos e Diretrizes para uma Educação Inclusiva: Revisão de Literatura. Revista Educação Especial, Santa Maria, p. 281-294, maio 2015. ISSN 1984-686X.

ProDeaf. O que é o ProDeaf? Disponível em: https://www.prodeaf.net//. Acessado em: Julho de 2018.

\footnotetext{
${ }^{7}$ SIMROAA é um Sistema de Recomendação Multiagente para Recomendar Objetos de Aprendizagem Acessíveis, projetado no âmbito do Projeto MIDOAA.
} 